Article

\title{
Analysis of Robot Selection Based on 2-Tuple Picture Fuzzy Linguistic Aggregation Operators
}

\author{
Arshad Ahmad Khan ${ }^{1}$, Muhammad Qiyas ${ }^{2}\left(\mathcal{D}\right.$, Saleem Abdullah ${ }^{2, *}$, Jianchao Luo ${ }^{1, *}(\mathbb{C})$ and \\ Mahwish Bano $^{3}$ \\ 1 College of Economics and Management, Northwest A\&F University, Yangling 712100, China; \\ arshadkhan@nwafu.edu.cn \\ 2 Department of Mathematics, Abdul Wali Khan University, Mardan 23200, Pakistan; \\ muhammadqiyas@awkum.edu.pk \\ 3 Department of Mathematics, Air University, Islamabad 44000, Pakistan; mahwish@mail.au.edu.pk \\ * Correspondence: jchluo@nwsuaf.edu.cn (J.L); saleemabdullah@awkum.edu.pk (S.A.)
}

Received: 12 September 2019; Accepted: 12 October 2019; Published: 21 October 2019

\begin{abstract}
The aim of this article is to propose the 2-tuple picture fuzzy linguistic aggregation operators and a decision-making model to deal with uncertainties in the form of 2-tuple picture fuzzy linguistic sets; 2-tuple picture fuzzy linguistic operators have more flexibility than general fuzzy set. We proposed a number of aggregation operators, namely, 2-TPFLWA, 2-TPFLOWA, 2-TPFLHA, 2-TPFLWG, 2-TPFLOWG, and 2-TPFLHG operators. The distinguished feature of the developed operators are studied. At that point, we used these operators to design a model to deal with multiple attribute decision-making issues under the 2-tuple picture fuzzy linguistic information. Then, a practical application of robot selection by manufacturing unit is given to prove the introduced technique and to show its practicability and effectiveness. Besides this, a systematic comparison analysis with other existent approaches is conducted to reveal the advantage of our developed method. Results indicate that the proposed method is suitable and effective for decision-making problems.
\end{abstract}

Keywords: picture fuzzy set; 2-tuple linguistic model; 2-tulpe picture fuzzy linguistic set

\section{Introduction}

Generally, there exists some uncertainty in the representation of data information during the decision-making (DM) process. To overcome this drawback, first, Zadeh [1] defined fuzzy set (FS). In fuzzy set, Zadeh only showed the positive membership grade of a number in the defined set, and applied it to many other fields, i.e., fuzzy decision-making problems [2,3]. However, there was no discussion for the negative membership grade. Therefore, due to this non-membership grade, the fuzzy set theory failed to solve the complete uncertainty in real-life problem. For this purpose, Atanassov [4] defined the concept of intuitionistic fuzzy set (IFS), which consists of positive and negative membership grades; IFS has the advantage of two memberships, which diminish the fuzziness. Garg [5,6] displayed generalized improved interactive aggregation operators to solve a decision-making problem under the IF set condition. Kaur and Garg [7] introduced cubic intuitionistic fuzzy set aggregation operator. Later, Kaur \& Garg [8] developed generalized aggregation operators with the cubic IF set information using the t-norm operations. Aside from them, different scholars (Garg \& Arora [9]; Garg \& Rani [10]; Shen \& Wang [11]; Peng, \& Wang [12]; Hoskova \& Maturo [13]; Collan \& Kacprzyk [14]) incorporated the idea of aggregation process in different applications and also given their related decision-making algorithm with the IF set and their expansion.

Atanassov discussed only two categories of responses i.e., "yes" and "no", but we have three types of responses in case of selection, for example, "yes", "no", and "refusal", and the complicated 
answer is "refusal". Therefore, to overcome this drawback, Cuong $[15,16]$ introduced a novel notion of picture fuzzy set (PFS), which dignified in three different functions, presenting the positive, neutral, and negative membership grades. Cuong [17] discussed some characteristic of PF sets and also approved their distance measures. Cuong and Hai [18] introduced fuzzy logic operators and defined fundamental operations for fuzzy derivation forms in the PF logic. Cuong, Kreinovich, and Ngan [19] examined the characteristic of picture fuzzy t-norm and t-conorm. Phong et al. [20] explored certain configuration of picture fuzzy relations. Wei et al. [21-23] defined many procedures to compute the closeness between PFSs. Presently, many researchers have developed more models in the PF sets condition: Correlation coefficients of PFS are proposed by Singh [24] and apply it to clustering analysis. Son et al. and Thong $[25,26]$ provided time arrangement calculation and temperature estimation on the basis of PFSs domain. Son $[27,28]$ defined PF as separation, distance and association measures, also combined them under the condition of PF sets. Van et al. [29] defined novel fuzzy derivation structure on PF set and improved classical fuzzy inference system. In [30,31], Thong et al. utilized the PF clustering approach for complex and particle clump optimization. Using the PF weighted cross-entropy concept, Wei [32] studied basic leadership technique and used this technique to rank the alternative. Based on PF sets, Yang et al. [33] defined flexible soft discernibly matrix of decision-making. In [34], Garg design aggregation of PF sets for MCDM problems. In [35], Peng et al. proposed picture fuzzy set approach and tested it in decision-making, also see [36-38] for the PF set. Ashraf et al. [39] extended the structure of cubic sets to the picture fuzzy sets. They also defined the notion of positive-internal, neutral-internal, negative-internal and positive-external, and neutral-external and negative-external cubic picture fuzzy sets.

Herrera \& Martinez [40] developed the concept of 2-tuple linguistic processing model based on the symbolic translation model, and also showed that the 2-tuple linguistic information processing manner can effectively avoid the loss and distortion of information. A group decision-making model was proposed by Herrera et al. [41], to manage a nonhomogeneous information. For choosing the appropriate agile manufacturing system, Wang [42] developed a 2-tuple fuzzy linguistic processing model. The TOPSIS technique is extended by Wei [43] for MAGDM problem with 2-tuple linguistic information. Chang \& Wen [44] proposed the efficient algorithm for DFMEA, combining the 2-tuple model and OWA operator. Bonferroni mean operators are extended by Jiang \& Wei [45] for the 2-tuple linguistic information. The dependent interval 2-tuple linguistic aggregation operators are developed by Li et al. [46] for MAGDM. Wang et al. [47] proposed an algorithm for the MAGDM problems, using the interval 2-tuple linguistic information and Choquet integral aggregation operators. To study the application of MAGDM on the supplier selection, Liu [48] defined the 2-tuple linguistic Muirhead mean operators. A consensus reaching model for the 2-tuple linguistic MAGDM was proposed by Zhang et al. [49], where the weight information are incomplete.

The remainder of the article is arranged as follows. In Section 2, we briefly discuss the basic knowledge about the picture fuzzy set and 2-tuple linguistic processing model. In Section 3, we define 2-TPFLS and their operational laws. In Section 4, we present some 2-tuple picture fuzzy linguistic averaging aggregation operators and discuss their basic properties. In Section 5, we present geometric aggregation operators of 2-tuple picture fuzzy linguistic number and their properties. Utilizing the 2TPFLWA and 2TPFLWG operators, we developed a model for multiattribute decision-making (MADM) problem and discussed the application of the developed approach and their comparison with existing approaches in Section 6. Finally, we write the conclusion in Section 7.

\section{Preliminaries}

In this section, we have presented some basic knowledge of the 2-tuple linguistic term set, picture fuzzy set, and 2-tuple picture fuzzy linguistic set.

Definition 1. Let $\hat{S}=\left(s_{1}, \ldots, s_{\tau}\right)$ be the collection of linguistic terms set and $\tau$ denote the odd cardinality [50,51], such that $s_{\tau}$, and $\tau$ represent the possible value of the linguistic variable and positive integer, respectively. 
Generally, $\tau$ is taken as 3,5 , etc., i.e., when $\tau=5$, then the linguistic term set $S$ is defined as $\left\{s_{1}=\right.$ Poor, $s_{2}=$ Slightly poor, $s_{3}=$ Fair, $s_{4}=$ Slightly good,$s_{5}=$ Good $\}$.

If $s_{\kappa}, s_{t} \in S$, then the following characteristic must be satisfied;

(1). The ordered of set as: $s_{\mathcal{K}} \prec s_{t}, \Leftrightarrow \kappa \prec t$;

(2). The operator of negation as: $\mathrm{Neg}\left(s_{\kappa}\right)=s_{\tau-\kappa}$;

(3). Maximum $\left(s_{\kappa}, s_{t}\right)=s_{\kappa}$, iff $s_{\mathcal{K}} \geq s_{t}$;

(4). Minimum $\left(s_{\kappa}, s_{t}\right)=s_{\kappa}$, iff $s_{\kappa} \leq s_{t}$.

Using the concept of symbolic translation, Herrera \& Martinez [50,51] introduced the 2-tuple fuzzy linguistic model. The model is used to denote the linguistic assessment information by means of a 2-tuple $\left(s_{l}, \alpha_{l}\right)$, and $s_{l}$ and $\alpha_{l}$ denote the linguistic label and symbolic translation, respectively, from the linguistic term set $S$ and $\alpha \in[-0.5,0.5]$.

Definition 2. Let $\beta$ be the result of an aggregation of the indices of a set of labels assessed in a linguistic term set $S[50,51]$, for example, the result of a symbolic aggregation operation, $\beta \in[1, \tau]$, where $\tau$ denotes the odd cardinality of $\dot{S}$. Let $\imath=\operatorname{round}(\beta)$ and $\alpha=\beta-\imath$ be two values, such that, $\imath \in[1, \tau]$ and $\alpha \in[-0.5,0.5]$, then $\alpha$ is said to be the symbolic translation.

Definition 3. Let $S=\left(s_{1}, \ldots, s_{\tau}\right)$ be the finite linguistic term set and $\beta \in[1, \tau]$ be the number value of the aggregation result of linguistic symbolic [50,51]. Then, the mapping $\Lambda$ are utilized to get the 2-tuple linguistic information equivalent to $\beta$, and defined as

$$
\begin{gathered}
\Lambda \quad: \quad[1, \tau] \rightarrow \dot{S} \times[-0.5,0.5), \\
\Lambda(\beta)=\left\{\begin{array}{c}
s_{l}, \imath=\operatorname{round}(\beta) \\
\alpha=\beta-\imath, \alpha \in[-0.5,0.5),
\end{array}\right.
\end{gathered}
$$

where round (.), $s_{1}, \alpha$ denotes the usual round operation, closest index label to $\beta$ and the value of the symbolic translation, respectively.

Definition 4. Let $\dot{S}=\left(s_{1}, \ldots, s_{\tau}\right)$ be the finite linguistic term set and $\left(s_{l}, \alpha_{l}\right)$ be a 2-tuple [50,51]. Then, there exists a mapping $\Lambda^{-1}$, such that from a 2 -tuple $\left(s_{l}, \alpha_{l}\right)$ it returns its equivalent numerical value $\beta \in[1, \tau] \subset R$, which is

$$
\begin{gathered}
\Lambda^{-1}: \quad \dot{S} \times[-0.5,0.5) \rightarrow[1, \tau], \\
\Lambda^{-1}\left(s_{\imath}, \alpha\right)=\imath+\alpha=\beta,
\end{gathered}
$$

We observe from Definitions 2 and 3 that the conversion of a linguistic term into a linguistic 2-tuple consists of adding a value 0 as symbolic translation:

$$
\Lambda\left(s_{l}\right)=\left(s_{l}, 0\right) .
$$

Definition 5. Let $\mathbb{R} \neq \phi$, be a set [52]. Then, $\Im$ is called picture fuzzy set, defined as

$$
\Im=\left\{\left\langle\ddot{a}_{\Im}(r), \ddot{e}_{\Im}(r), \ddot{u}_{\Im}(r) \mid r \in \mathbb{R}\right\rangle\right\},
$$

where $\ddot{a}_{\Im}(r), \ddot{e}_{\Im}(r), \ddot{u}_{\Im}(r): \mathbb{R} \rightarrow[0,1]$ be the positive, neutral, and negative membership grades of each $r \in \mathbb{R}$, correspondingly. $\ddot{a}_{\Im}(r), \ddot{e}_{\Im}(r)$, and $\ddot{u}_{\Im}(r)$ satisfy that $0 \leq \ddot{a}_{\Im}(r)+\ddot{e}_{\Im}(r)+\ddot{u}_{\Im}(r) \leq 1 \forall r \in \mathbb{R}$. $\pi_{\Im}(r)=1-\ddot{a}_{\Im}(r)+\ddot{e}_{\Im}(r)+\ddot{u}_{\Im}(r)$ is the refusal grade of $r$ in $\mathbb{R}$, and a triple-component $\left\langle\ddot{a}_{\Im}, \ddot{e}_{\Im}, \ddot{u}_{\Im}\right\rangle$ is called the picture fuzzy number, and each picture fuzzy number is in the form of $E=\left\langle\ddot{a}_{\Im}, \ddot{e}_{\Im}, \ddot{u}_{\Im}\right\rangle$, where $\ddot{a}_{\Im}, \ddot{e}_{\Im}$, and $\ddot{u}_{\Im} \in[0,1]$, having the condition

$$
0 \leq \ddot{a}_{\Im}+\ddot{e}_{\Im}+\ddot{u}_{\Im} \leq 1 .
$$


Definition 6. Let $\Im_{1}=\left\langle\ddot{a}_{\Im_{1}}(r), \ddot{e}_{\Im_{1}}(r), \ddot{u}_{\Im_{1}}(r)\right\rangle$ and $\Im_{2}=\left\langle\ddot{a}_{\Im_{2}}(r), \ddot{e}_{\Im_{2}}(r), \ddot{u}_{\Im_{2}}(r)\right\rangle$ [52], which are two picture fuzzy numbers defined on the of discourse $\mathbb{R} \neq \phi$, some operations on picture fuzzy numbers are defined as follows.

(1). $\Im_{1} \subseteq \Im_{2}$ if

$$
\ddot{a}_{\Im_{1}}(r) \leq \ddot{a}_{\Im_{2}}(r), \ddot{e}_{\Im_{1}}(r) \leq \ddot{e}_{\Im_{2}}(r) \text { and } \ddot{u}_{\Im_{1}}(r) \geq \ddot{u}_{\Im_{2}}(r), \forall r \in \mathbb{R},
$$

(2). Union

$$
\Im_{1} \cup \Im_{2}=\left(\begin{array}{c}
\left(r, \max \left(\ddot{a}_{\Im_{1}}(r), \ddot{a}_{\Im_{2}}(r)\right), \min \left(\ddot{e}_{\Im_{1}}(r), \ddot{e}_{\Im_{2}}(r)\right),\right. \\
\min \left(\ddot{u}_{\Im_{1}}(r), \ddot{u}_{\Im_{2}}(r)\right) \mid r \in \mathbb{R}
\end{array}\right) ;
$$

(3). Intersection

$$
\Im_{1} \cap \Im_{2}=\left(\begin{array}{c}
\left(r, \min \left(\ddot{a}_{\Im_{1}}(r), \ddot{a}_{\Im_{2}}(r)\right), \min \left(\ddot{e}_{\Im_{1}}(r), \ddot{e}_{\Im_{2}}(r)\right),\right. \\
\max \left(\ddot{u}_{\Im_{1}}(r), \ddot{u}_{\Im_{2}}(r)\right) \mid r \in \mathbb{R}
\end{array}\right) ;
$$

(4). Compliment

$$
\Im_{1}^{c}=\left\{\left(r, \ddot{u}_{\Im_{1}}(r), \ddot{e}_{\Im_{1}}(r), \ddot{a}_{\Im_{1}}(r) \mid r \in \mathbb{R}\right\} .\right.
$$

Definition 7. Let $\Im_{1}=\left\langle\ddot{a}_{\Im_{1}}, \ddot{e}_{\Im_{1}}, \ddot{u}_{\Im_{1}}\right\rangle$ and $\Im_{2}=\left\langle\ddot{a}_{\Im_{2}}, \ddot{\Im}_{\Im_{2}}, \ddot{u}_{\Im_{2}}\right\rangle$ [53], which are two picture fuzzy numbers defined on the universe of discourse $\mathbb{R} \neq \phi$; some operations on picture fuzzy numbers are defined as follows, with $\lambda \geq 0$.

1. $\Im_{1} \oplus \Im_{2}=\left\{\ddot{a}_{\Im_{1}}^{2}+\ddot{a}_{\Im_{2}}^{2}-\ddot{a}_{\Im_{1}}^{2} \cdot \ddot{a}_{\Im_{2}}^{2}, \ddot{e}_{\Im_{1}} \ddot{e}_{\Im_{2}}, \ddot{u}_{\Im_{1}} \cdot \ddot{u}_{\Im_{2}}\right\} ;$

2. $\Im_{1} \otimes \Im_{2}=\left\{\ddot{a}_{\Im_{1}} . \ddot{a}_{\Im_{2}}, \ddot{e}_{\Im_{1}}^{2}+\ddot{e}_{\Im_{2}}^{2}-\ddot{e}_{\Im_{1}}^{2} . \ddot{e}_{\Im_{2}{ }^{\prime}}^{2}, \ddot{u}_{\Im_{1}}^{2}+\ddot{u}_{\Im_{2}}^{2}-\ddot{u}_{\Im_{1}}^{2} . \ddot{u}_{\Im_{2}}^{2}\right\}$;

3. $\lambda \cdot \Im_{1}=\left\{1-\left(1-\ddot{a}_{\Im_{1}}^{2}\right)^{\lambda},\left(\ddot{e}_{\Im_{1}}\right)^{\lambda},\left(\ddot{u}_{\Im_{1}}\right)^{\lambda}\right\}$;

4. $\quad \Im_{1}^{\lambda}=\left\{\left(\ddot{a}_{\Im_{1}}\right)^{\lambda}, 1-\left(1-\ddot{e}_{\Im_{1}}^{2}\right)^{\lambda}, 1-\left(1-\ddot{u}_{\Im_{1}}^{2}\right)^{\lambda}\right\}$;

\section{2-Tuple Picture Fuzzy Linguistic Sets}

In the following, we introduced the concept of 2-tuple picture fuzzy linguistic sets and their basic operations based on the picture fuzzy set and 2-tuple linguistic information.

Definition 8. A 2-tuple picture fuzzy linguistic set $\Im$ in $\mathbb{R} \neq \phi$ is defined as

$$
\Im=\left\{\left\langle s_{\ddot{a}(r)}, s_{\ddot{e}(r)}, s_{\ddot{u}(r)} \mid r \in \mathbb{R}\right\rangle\right\},
$$

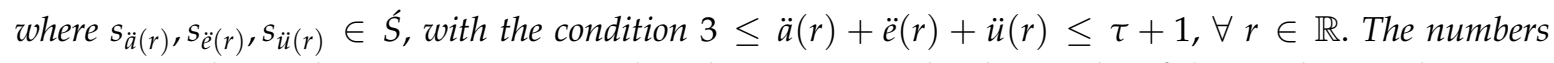
$s_{\ddot{u}(r)}, s_{\ddot{e}(r)}$ and $s_{\ddot{u}(r)}$ denote positive, neutral, and negative membership grades of the number $r$ to linguistic variable $\Im$. The term $s_{\chi(r)}$ is said to be the refusal grade of the element $r$ to linguistic variable $\Im$, and is defined as $s_{\chi(r)}=s_{\tau+1-\ddot{a}(r)-\ddot{e}(r)-\ddot{u}(r)}$.

For convenience, we said $\widetilde{\Im}=\left\langle\left(s_{\ddot{a}(r)}, \alpha\right),\left(s_{\ddot{e}(r)}, \beta\right),\left(s_{\ddot{u}(r)}, \gamma\right)\right\rangle$, a 2-tuple picture fuzzy linguistic number (2TPFLN), where $s_{\ddot{a}(r)}, s_{\ddot{e}(r)}, s_{\ddot{u}(r)} \in S, 3 \leq \Lambda^{-1}\left(s_{\ddot{a}(r)}, \alpha\right)+\Lambda^{-1}\left(s_{\ddot{e}(r)}, \beta\right)+\Lambda^{-1}\left(s_{\ddot{u}(r)}, \gamma\right) \leq$ $\tau+1$, and $\alpha, \beta, \gamma \in[-0.5,0.5)$.

Definition 9. Let $\phi, \varphi$, and $\chi$ be the results of the aggregation of the indices of a set of labels assessed in a linguistic term set, $S$; for example, the result of a symbolic aggregation operation, $\phi, \varphi, \chi \in[1, \tau], 3 \leq \phi+\varphi+$ $\chi \leq \tau+1$, (where $\tau$ be the cardinality of $\hat{S})$. Assume that $\ddot{a}=\operatorname{round}(\phi), \ddot{e}=\operatorname{round}(\varphi), \ddot{u}=\operatorname{round}(\chi)$ and that $\alpha=\phi-\ddot{a}, \beta=\varphi-\ddot{e}, \gamma=\chi-\ddot{u}$ are the six values, such that $\phi, \varphi, \chi \in[1, \tau]$ and $\alpha, \beta, \gamma \in[-0.5,0.5)$, then $\alpha, \beta$, and $\gamma$ are called symbolic translations. 
Definition 10. Let $S=\left(s_{1}, \ldots, s_{\tau}\right)$ be the linguistic set and $\phi, \varphi, \chi \in[1, \tau]$ be the three-number value, denoting the aggregation result of linguistic symbolic. Then, a mapping $\Lambda$ is utilized to get the 2-tuple linguistic information equivalent to $\phi, \varphi$, and $\chi$, and is defined as

$$
\begin{gathered}
\Lambda: \quad[1, \tau] \rightarrow \dot{S} \times[-0.5,0.5), \\
\Lambda(\phi)=\left\{\begin{array}{c}
s_{\ddot{a}}, \ddot{a}=\operatorname{round}(\phi) \\
\alpha=\phi-\ddot{a}, \alpha \in[-0.5,0.5),
\end{array}\right. \\
\Lambda(\varphi)=\left\{\begin{array}{c}
s_{\ddot{e},}, \ddot{e}=\operatorname{round}(\varphi) \\
\beta=\varphi-\ddot{e}, \beta \in[-0.5,0.5),
\end{array}\right. \\
\Lambda(\gamma)=\left\{\begin{array}{c}
s_{\ddot{u}}, \ddot{u}=\operatorname{round}(\chi) \\
\gamma=\chi-\ddot{u}, \gamma \in[-0.5,0.5),
\end{array}\right.
\end{gathered}
$$

where round (.), $s_{\ddot{a},}, s_{\ddot{e}}$, and $s_{\ddot{u}}$ and $\alpha, \beta$, and $\gamma$ denoted the usual round operation; closest index label to $\phi, \varphi$, and $\chi$; and the value of the symbolic translation, respectively.

Definition 11. Let $\dot{S}=\left(s_{1}, \ldots, s_{\tau}\right)$ is the finite linguistic term set and $\widetilde{\Im}=\left\langle\left(s_{\ddot{a}}, \alpha\right),\left(s_{\ddot{e}}, \beta\right),\left(s_{\ddot{u}}, \gamma\right)\right\rangle$, be a 2-tuple picture fuzzy linguistic number (2TPFLN). Then, there exists a mapping $\Lambda^{-1}$, such that from a 2-tuple picture fuzzy numbers $\left\langle\left(s_{\ddot{a}}, \alpha\right),\left(s_{\ddot{e}}, \beta\right),\left(s_{\ddot{u}}, \gamma\right)\right\rangle$ and it returns its equivalent numerical value $\phi, \varphi, \chi \in[1, \tau] \subset$ $R$, which is

$$
\begin{gathered}
\Lambda^{-1}: \quad \dot{S} \times[-0.5,0.5) \rightarrow[1, \tau], \\
\Lambda^{-1}\left(s_{\ddot{a}}, \alpha\right)=\ddot{a}+\alpha=\phi, \\
\Lambda^{-1}\left(s_{\ddot{e}}, \beta\right)=\ddot{e}+\beta=\varphi, \\
\Lambda^{-1}\left(s_{\ddot{u}}, \gamma\right)=\ddot{u}+\gamma=\chi .
\end{gathered}
$$

From Definitions 2 and 3, we observe that the conversion of a linguistic term into a linguistic 2-tuple consists of adding a value 0 as symbolic translation:

$$
\Lambda\left\langle s_{\ddot{u}(r)}, s_{\ddot{e}(r)}, s_{\ddot{u}(r)}\right\rangle=\left\langle\left(s_{\ddot{u}(r)}, 0\right),\left(s_{\ddot{e}(r)}, 0\right),\left(s_{\ddot{u}(r), 0)\rangle .}\right.\right.
$$

Definition 12. Let $\widetilde{\Im}=\left\langle\left(s_{\ddot{a}}, \alpha\right),\left(s_{\ddot{e}}, \beta\right),\left(s_{\ddot{u}}, \gamma\right)\right\rangle$, a 2-tuple picture fuzzy linguistic number (2TPFLN). Then, the score function of 2 TPFLN is as follows,

$$
\operatorname{Sc}(\widetilde{\Im})=\Lambda\left(\frac{\tau+\Lambda^{-1}\left(s_{\ddot{u}}, \alpha\right)-\Lambda^{-1}\left(s_{\ddot{e}}, \beta\right)-\Lambda^{-1}\left(s_{\ddot{u}}, \gamma\right)}{3}\right), \Lambda^{-1}(\operatorname{Sc}(\widetilde{\Im})) \in[1, \tau]
$$

Definition 13. Let $\widetilde{\Im}=\left\langle\left(s_{\ddot{a}}, \alpha\right),\left(s_{\ddot{e}}, \beta\right),\left(s_{\ddot{u}}, \gamma\right)\right\rangle$, a 2-tuple picture fuzzy linguistic number (2TPFLN). Then, the accuracy function of 2TPFLN is as follows,

$$
H c(\widetilde{\Im})=\Lambda\left(\frac{\Lambda^{-1}\left(s_{\ddot{u}}, \alpha\right)+\Lambda^{-1}\left(s_{\ddot{e}}, \beta\right)+\Lambda^{-1}\left(s_{\ddot{u}}, \gamma\right)}{3}\right), \Lambda^{-1}(H c(\widetilde{\Im})) \in[1, \tau]
$$

Definition 14. Let $\widetilde{\Im}_{1}=\left\langle\left(s_{\ddot{a}_{1}}, \alpha_{1}\right),\left(s_{\ddot{e}_{1}}, \beta_{1}\right),\left(s_{\ddot{u}_{1}}, \gamma_{1}\right)\right\rangle$ and $\widetilde{\Im}_{2}=\left\langle\left(s_{\ddot{u}_{2}}, \alpha_{2}\right),\left(s_{\ddot{e}_{2}}, \beta_{2}\right),\left(s_{\ddot{u}_{2}}, \gamma_{2}\right)\right\rangle$ be the 2-tuple picture fuzzy linguistic numbers (2TPFLNs). Then, using Definitions 12 and 13, the equating technique can be described as

(1) If $\operatorname{Sc}\left(\widetilde{\Im}_{1}\right)>\operatorname{Sc}\left(\widetilde{\Im}_{2}\right)$, then $\widetilde{\Im}_{1}$ is grater than $\widetilde{\Im}_{2}$,

(2) If $S c\left(\widetilde{\Im}_{1}\right)=\operatorname{Sc}\left(\widetilde{\Im}_{2}\right)$, then

(3) If $H c\left(\widetilde{\Im}_{1}\right)>H c\left(\widetilde{\Im}_{2}\right)$, then $\widetilde{\Im}_{1}$ is grater than $\widetilde{\Im}_{2}$,

(4) If $\mathrm{Hc}\left(\widetilde{\Im}_{1}\right)=H c\left(\widetilde{\Im}_{2}\right)$, then $\widetilde{\Im}_{1}$ and $\widetilde{\Im}_{2}$ have the same information. 
Definition 15. Let $\widetilde{\Im}_{1}=\left\langle\left(s_{\ddot{a}_{1}}, \alpha_{1}\right),\left(s_{\ddot{e}_{1}}, \beta_{1}\right),\left(s_{\ddot{u}_{1}}, \gamma_{1}\right)\right\rangle$ and $\widetilde{\Im}_{2}=\left\langle\left(s_{\ddot{u}_{2}}, \alpha_{2}\right),\left(s_{\ddot{e}_{2}}, \beta_{2}\right),\left(s_{\ddot{u}_{2}}, \gamma_{2}\right)\right\rangle$ be the two 2-tuple picture fuzzy linguistic number (2TPFLN). Then,

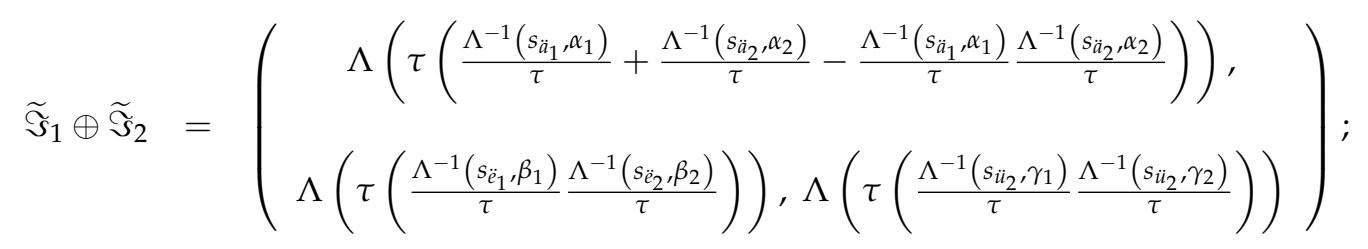

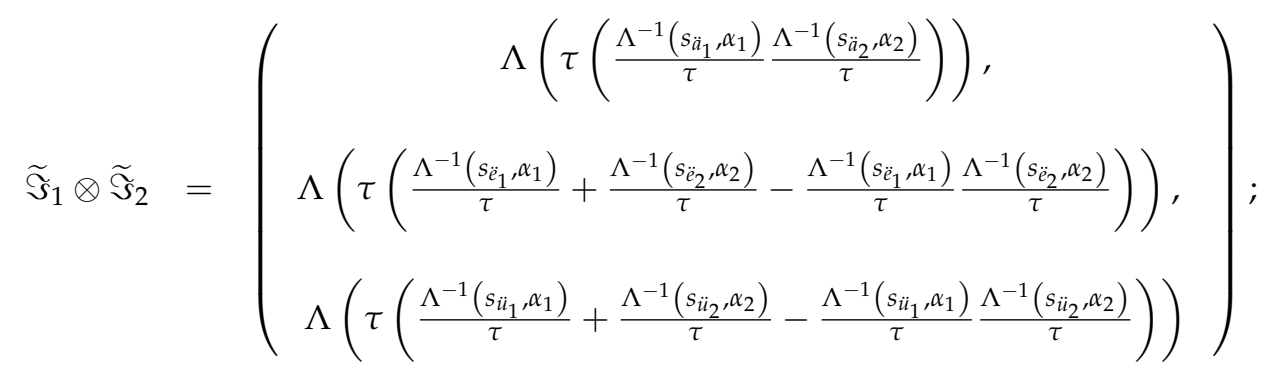

$$
\begin{aligned}
& \lambda \widetilde{\Im}_{1}=\left(\begin{array}{c}
\Lambda\left(\tau\left(1-\left(1-\frac{\Lambda^{-1}\left(s_{\ddot{a}_{1}}, \alpha_{1}\right)}{\tau}\right)^{\lambda}\right)\right), \Lambda\left(\tau\left(\frac{\Lambda^{-1}\left(s_{\tilde{e}_{1}}, \alpha_{1}\right)}{\tau}\right)^{\lambda}\right), \\
\Lambda\left(\tau\left(\frac{\Lambda^{-1}\left(s_{i_{1}}, \alpha_{1}\right)}{\tau}\right)^{\lambda}\right)
\end{array}\right) ; \\
& \left(\widetilde{\Im}_{1}\right)^{\lambda}=\left(\begin{array}{c}
\Lambda\left(\tau\left(\frac{\Lambda^{-1}\left(s_{\ddot{a}_{1}}, \alpha_{1}\right)}{\tau}\right)^{\lambda}\right), \Lambda\left(\tau\left(1-\left(1-\frac{\Lambda^{-1}\left(s_{\ddot{e}_{1}}, \alpha_{1}\right)}{\tau}\right)^{\lambda}\right)\right) \\
\Lambda\left(\tau\left(1-\left(1-\frac{\Lambda^{-1}\left(s_{\dot{u}_{1}}, \alpha_{1}\right)}{\tau}\right)^{\lambda}\right)\right)
\end{array}\right) .
\end{aligned}
$$

\section{Averaging Aggregation Operators on 2-Tuple Picture Fuzzy Linguistic Numbers}

In this section, we used the information of 2-tuple picture fuzzy linguistic numbers, and developed some averaging aggregation operators and discussed their properties.

Definition 16. Let $\widetilde{\Im}=\left\langle\left(s_{\ddot{a}}, \alpha\right),\left(s_{\ddot{e}}, \beta\right),\left(s_{\ddot{u}}, \gamma\right)\right\rangle(j=1, \ldots, n)$ be the set of 2-tuple picture fuzzy linguistic numbers. Then, the function $\Omega^{n} \rightarrow \Omega$ is a 2-tuple picture fuzzy linguistic weighted average (2TPFLWA) operator, defined as

$$
2 \operatorname{TPFLWA} A_{\Theta}\left(\widetilde{\Im}_{1}, \ldots, \widetilde{\Im}_{n}\right)=\bigoplus_{j=1}^{n}\left(\Theta_{j} \widetilde{\Im}_{j}\right),
$$

where $\Theta=\left(\Theta_{1}, \ldots, \Theta_{n}\right)^{T}$ is the weighting vector of $\widetilde{\Im}_{j}$, and $\Theta_{j}>0, \sum_{j=1}^{n} \Theta_{j}=1$. 
Theorem 1. The aggregated value by using the 2TPFLWA operator is also a 2-tuple picture fuzzy linguistic numbers, such that

$$
\begin{aligned}
& 2 \operatorname{TPFLWA} A_{\Theta}\left(\widetilde{\Im}_{1}, \ldots, \widetilde{\Im}_{n}\right)=\bigoplus_{j=1}^{n}\left(\Theta_{j} \widetilde{\Im}_{j}\right)
\end{aligned}
$$

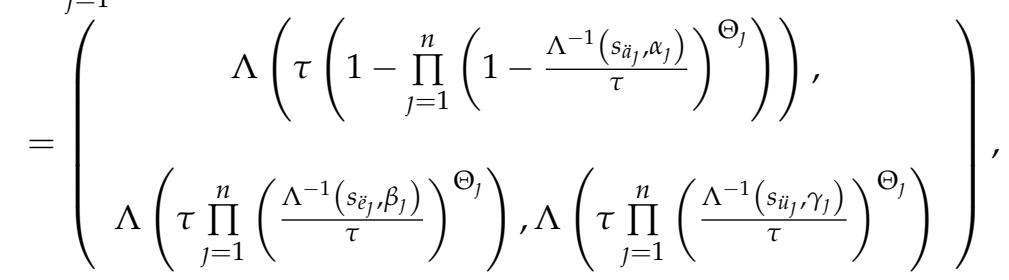

where $\Theta=\left(\Theta_{1}, \ldots, \Theta_{n}\right)^{T}$ is the weights of $\widetilde{\Im}_{j}$, and $\Theta_{j}>0, \sum_{j=1}^{n} \Theta_{j}=1$.

Proof. We used the mathematical induction principle to prove Equation (21).

(1). When $n=2$, we get

$$
\begin{gathered}
\Theta_{1} \widetilde{\Im}_{1}=\left(\begin{array}{c}
\Lambda\left(\tau\left(1-\left(1-\frac{\Lambda^{-1}\left(s_{\ddot{u}_{1}}, \alpha_{1}\right)}{\tau}\right)^{\Theta_{1}}\right)\right), \\
\Lambda\left(\tau\left(\frac{\Lambda^{-1}\left(s_{\ddot{e}_{1},}, \beta_{1}\right)}{\tau}\right)^{\Theta_{1}}\right), \Lambda\left(\tau\left(\frac{\Lambda^{-1}\left(s_{\ddot{u}_{1},}, \gamma_{1}\right)}{\tau}\right)^{\Theta_{1}}\right)
\end{array}\right) . \\
\Theta_{2} \widetilde{\Im}_{2}=\left(\begin{array}{c}
\Lambda\left(\tau\left(1-\left(1-\frac{\Lambda^{-1}\left(s_{\ddot{u}_{2}}, \alpha_{2}\right)}{\tau}\right)^{\Theta_{2}}\right)\right), \\
\Lambda\left(\tau\left(\frac{\Lambda^{-1}\left(s_{\ddot{e}_{1},}, \beta_{2}\right)}{\tau}\right)^{\Theta_{2}}\right), \Lambda\left(\tau\left(\frac{\Lambda^{-1}\left(s_{\ddot{u}_{2},}, \gamma_{2}\right)}{\tau}\right)^{\Theta_{2}}\right)
\end{array}\right) .
\end{gathered}
$$

Then,

$$
\begin{aligned}
& 2 \operatorname{TPFLWA} A_{\Theta}\left(\widetilde{\Im}_{1}, \widetilde{\Im}_{2}\right)=\left(\Theta_{1} \widetilde{\Im}_{1} \oplus \Theta_{2} \widetilde{\Im}_{2}\right) \\
& =\left(\begin{array}{c}
\Lambda\left(\tau\left(1-\left(1-\frac{\Lambda^{-1}\left(s_{\ddot{p}_{1},}, \alpha_{1}\right)}{\tau}\right)^{\Theta_{1}}\left(1-\frac{\Lambda^{-1}\left(s_{\ddot{\theta}_{2},}, \alpha_{2}\right)}{\tau}\right){ }^{\Theta_{2}}\right)\right) \\
\Lambda\left(\tau\left(\frac{\Lambda^{-1}\left(s_{\ddot{e}_{1},}, \beta_{1}\right)}{\tau}\right)^{\Theta_{1}}\left(\frac{\Lambda^{-1}\left(s_{\ddot{e}_{2},}, \beta_{2}\right)}{\tau}\right)^{\Theta_{2}}\right), \\
\Lambda\left(\tau\left(\frac{\Lambda^{-1}\left(s_{\ddot{u}_{1},}, \gamma_{1}\right)}{\tau}\right)^{\Theta_{1}}\left(\frac{\Lambda^{-1}\left(s_{\ddot{u}_{2}}, \gamma_{2}\right)}{\tau}\right)^{\Theta_{2}}\right)
\end{array}\right.
\end{aligned}
$$


(2). Let Equation (21) is true for $n=\kappa$; that is,

$$
\begin{aligned}
& 2 \operatorname{TPFLWA} A_{\Theta}\left(\widetilde{\Im}_{1}, \ldots, \widetilde{\Im}_{\kappa}\right)=\bigoplus_{j=1}^{\kappa}\left(\Theta_{j} \widetilde{\Im}_{j}\right)
\end{aligned}
$$

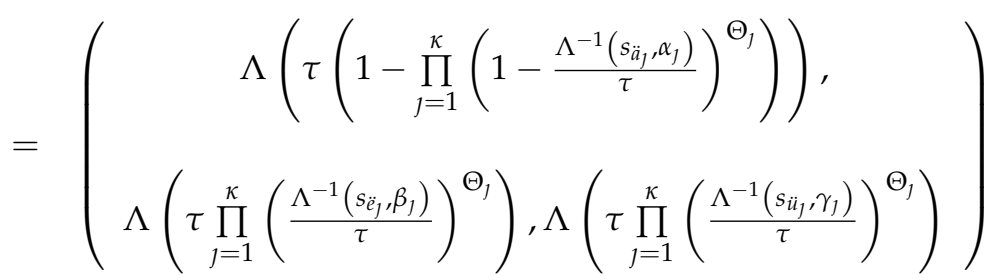

and we prove Equation (21), for $n=\kappa+1$. Then,

$$
\begin{aligned}
& 2 T P F L W A_{\Theta}\left(\widetilde{\Im}_{1}, \ldots, \widetilde{\Im}_{\kappa+1}\right)=\Theta_{1} \widetilde{\Im}_{1} \oplus \Theta_{2} \widetilde{\Im}_{2} \oplus \ldots \oplus \Theta_{\kappa} \widetilde{\Im}_{\kappa} \oplus \Theta_{\kappa+1} \widetilde{\Im}_{\kappa+1}
\end{aligned}
$$

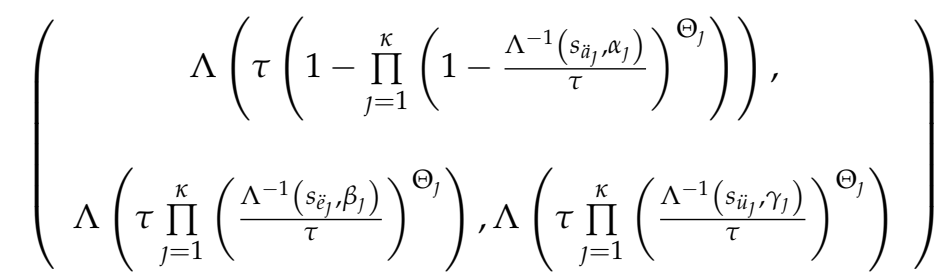

$$
\begin{aligned}
& =\left(\begin{array}{c}
\Lambda\left(\tau\left(1-\left(1-\frac{\Lambda^{-1}\left(s_{\ddot{t}_{\kappa+1}}, \alpha_{\kappa+1}\right)}{\tau}\right)^{\Theta_{\kappa+1}}\right)\right), \\
\Lambda\left(\tau\left(\frac{\Lambda^{-1}\left(s_{\tilde{e}_{\kappa+1}}, \beta_{\kappa+1}\right)}{\tau}\right)^{\Theta_{\kappa+1}}\right), \Lambda\left(\tau\left(\frac{\Lambda^{-1}\left(s_{u_{\kappa+1}}, \gamma_{\kappa+1}\right)}{\tau}\right)^{\Theta_{\kappa+1}}\right)
\end{array}\right)
\end{aligned}
$$

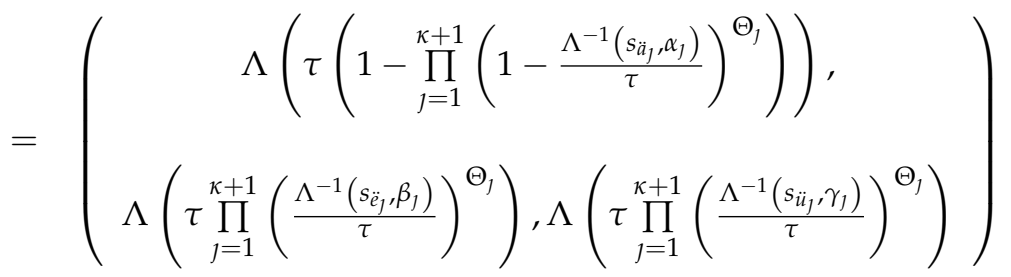

which shows that the aggregated value is also a 2TPFLN. Therefore, Equation (21) holds for all $n$.

Property 1 (Idempotency). If $\widetilde{\Im}_{j}=\widetilde{\Im}$ for all j, then

$$
2 \operatorname{TPFLWA}\left(\widetilde{\Im}_{1}, \ldots, \widetilde{\Im}_{n}\right)=\widetilde{\Im} .
$$


Proof. As $\widetilde{\Im}_{j}=\widetilde{\Im}(j=1, \ldots, n)$, the WA aggregation result of 2 TPFLNs can be calculated using

$$
\begin{aligned}
& 2 \operatorname{TPFLWA} A_{\Theta}\left(\widetilde{\Im}_{1}, \ldots, \widetilde{\Im}_{n}\right)=\bigoplus_{j=1}^{n}\left(\Theta_{j} \widetilde{\Im}_{j}\right) \\
& =\left(\begin{array}{c}
\Lambda\left(\tau\left(1-\prod_{j=1}^{n}\left(1-\frac{\Lambda^{-1}\left(s_{\ddot{s}_{j},}, \alpha_{\jmath}\right)}{\tau}\right)^{\Theta_{\jmath}}\right)\right), \\
\Lambda\left(\tau \prod_{j=1}^{n}\left(\frac{\Lambda^{-1}\left(s_{\ddot{e}_{j}, \beta_{\jmath}}\right)}{\tau}\right)^{\Theta_{\jmath}}\right), \Lambda\left(\tau \prod_{j=1}^{n}\left(\frac{\Lambda^{-1}\left(s_{i_{j},}, \gamma_{\jmath}\right)}{\tau}\right)^{\Theta_{\jmath}}\right)
\end{array}\right),
\end{aligned}
$$

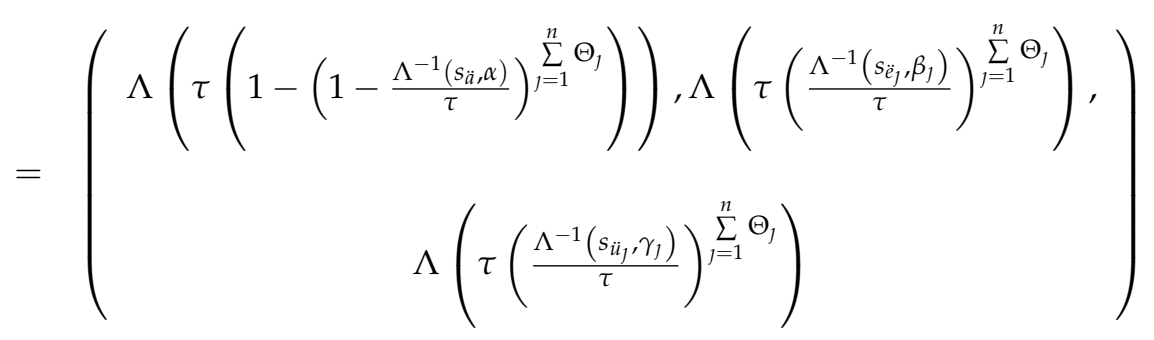

$$
\begin{aligned}
& =\left(\begin{array}{c}
\Lambda\left(\tau\left(1-\left(1-\frac{\Lambda^{-1}\left(s_{\tilde{a}}, \alpha\right)}{\tau}\right)\right)\right), \Lambda\left(\tau\left(\frac{\Lambda^{-1}\left(s_{\tilde{e}}, \beta\right)}{\tau}\right)\right) \\
\Lambda\left(\tau\left(\frac{\Lambda^{-1}\left(s_{\tilde{u}}, \gamma\right)}{\tau}\right)\right)
\end{array}\right) \\
& =\left\langle\left(s_{\ddot{a}}, \alpha\right),\left(s_{\ddot{e}}, \beta\right),\left(s_{\ddot{u}}, \gamma\right)\right\rangle=\widetilde{\Im}
\end{aligned}
$$

Property 2 (Boundedness). Let $\widetilde{\Im}_{j}(j=1, \ldots, n)$ be a set of 2 TPFLNs, $\widetilde{\Im}^{+}=\max _{j} \widetilde{\Im}_{j}$, and $\widetilde{\Im}^{-}=\min _{j} \widetilde{\Im}_{j}$, then

$$
\widetilde{\Im}^{-} \leq 2 \operatorname{TPFLWA}\left(\widetilde{\Im}_{1}, \ldots, \widetilde{\Im}_{n}\right) \leq \widetilde{\Im}^{+}
$$

Proof. As $\widetilde{\Im}^{-}$are the minimum 2TPFLNs and $\widetilde{\Im}^{+}$are the maximum 2TPFLNs, there exists $\widetilde{\Im}^{-} \leq \widetilde{\Im}_{j} \leq$ $\widetilde{\Im}^{+}$. Thus, we have

$$
\sum_{j=1}^{n} \Theta_{j} \widetilde{\Im}^{-} \leq \sum_{j=1}^{n} \Theta_{j} \widetilde{\Im} \leq \sum_{j=1}^{n} \Theta_{j} \widetilde{\Im}^{+}
$$

Corresponding to the property (1), there are $\sum_{j=1}^{n} \Theta_{j} \widetilde{\Im}^{-}=\widetilde{\Im}^{-}$and $\sum_{j=1}^{n} \Theta_{j} \widetilde{\Im}^{+}=\widetilde{\Im}^{+}$. Therefore,

$$
\widetilde{\Im}^{-} \leq 2 \operatorname{TPFLWA}\left(\widetilde{\Im}_{1}, \ldots, \widetilde{\Im}_{n}\right) \leq \widetilde{\Im}^{+}
$$

Property 3 (Monotonicity). Let $\widetilde{\Im}_{j}(j=1, \ldots, n)$ and $\widetilde{\Im}_{j}^{\prime}(j=1, \ldots, n)$ be a collection of 2 TPFLNs, if $\widetilde{\Im}_{j} \leq$ $\widetilde{\Im}_{j}^{\prime}, \forall j$, then

$$
2 \operatorname{TPFLWA}\left(\widetilde{\Im}_{1}, \ldots, \widetilde{\Im}_{n}\right) \leq 2 \operatorname{TPFLWA}\left(\widetilde{\Im}_{1}^{\prime}, \ldots, \widetilde{\Im}_{n}^{\prime}\right) .
$$


Proof. As $\widetilde{\Im}_{j} \leq \widetilde{\Im}_{j}^{\prime}(j=1, \ldots, n)$, there exist $\sum_{j=1}^{n} \Theta_{j} \widetilde{\Im} \leq \sum_{j=1}^{n} \Theta_{j} \widetilde{\Im}^{\prime}$. Therefore,

$$
2 T P F L W A_{\Theta}\left(\widetilde{\Im}_{1}, \ldots, \widetilde{\Im}_{n}\right) \leq 2 T P F L W A_{\Theta}\left(\widetilde{\Im}_{1}^{\prime}, \ldots, \widetilde{\Im}_{n}^{\prime}\right) .
$$

Definition 17. Let $\widetilde{\Im}=\left\langle\left(s_{\ddot{a}}, \alpha\right),\left(s_{\ddot{e}}, \beta\right),\left(s_{\ddot{u}}, \gamma\right)\right\rangle(j=1, \ldots, n)$ be the set of 2-tuple picture fuzzy linguistic numbers. Then, the 2-tuple picture fuzzy linguistic ordered weighted averaging (2TPFLOWA) operator is a function $\Omega^{n} \rightarrow \Omega$, with $\Theta=\left(\Theta_{1}, \ldots, \Theta_{n}\right)^{T}$ as the associated weights and $\Theta_{j}>0, \sum_{j=1}^{n} \Theta_{j}=1$. Then,

$$
\begin{aligned}
& 2 \operatorname{TPFLOWA} A_{\Theta}\left(\widetilde{\Im}_{1}, \ldots, \widetilde{\Im}_{n}\right)=\bigoplus_{j=1}^{n}\left(\Theta_{j} \widetilde{\Im}_{\sigma(\jmath)}\right)
\end{aligned}
$$

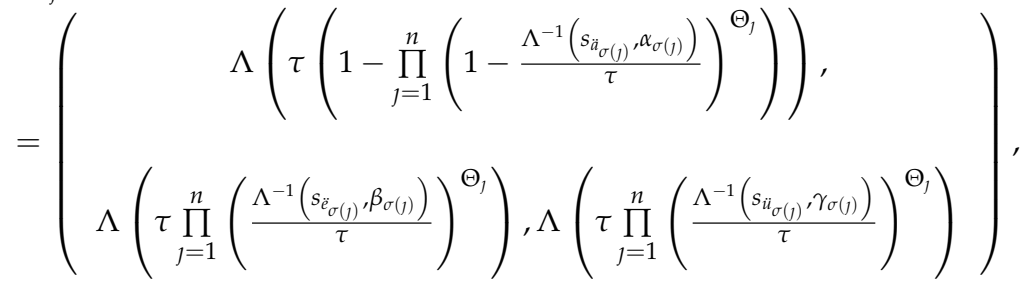

where the permutation of $(1, \ldots, n)$ are $\left(\sigma(1), \ldots, \sigma(n)\right.$, and defined as $\widetilde{\Im}_{\sigma(\jmath-1)} \geq \widetilde{\Im}_{\sigma(\jmath)} \forall j=2, \ldots, n$.

Definition 18. Let $\widetilde{\Im}=\left\langle\left(s_{\ddot{a}}, \alpha\right),\left(s_{\ddot{e}}, \beta\right),\left(s_{\ddot{u}}, \gamma\right)\right\rangle(j=1, \ldots, n)$ be the set of 2-tuple picture fuzzy linguistic numbers. Then, the 2-tuple picture fuzzy linguistic hybrid average (2TPFLHA) operator is a function of $\Omega^{n} \rightarrow \Omega$, such that

$$
\begin{aligned}
& 2 \operatorname{TPFLHA} A_{\Theta}\left(\widetilde{\Im}_{1}, \ldots, \widetilde{\Im}_{n}\right)=\bigoplus_{j=1}^{n}\left(\Theta_{j} \underset{\Im_{\sigma(\jmath)}}{\stackrel{*}{\Im^{\prime}}}\right)
\end{aligned}
$$

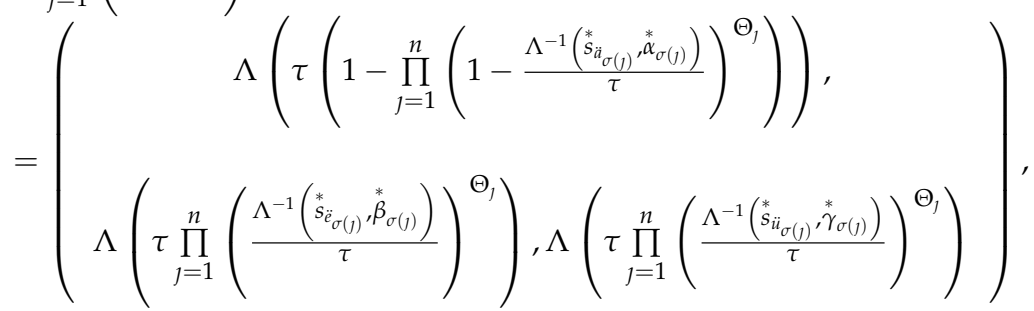

where $\Theta=\left(\Theta_{1}, \ldots, \Theta_{n}\right)^{T}$ denote the associated weights, such that $\Theta_{j}>0, \sum_{j=1}^{n} \Theta_{j}=1 ; \stackrel{*}{\Im}_{\sigma(j)}$ is the

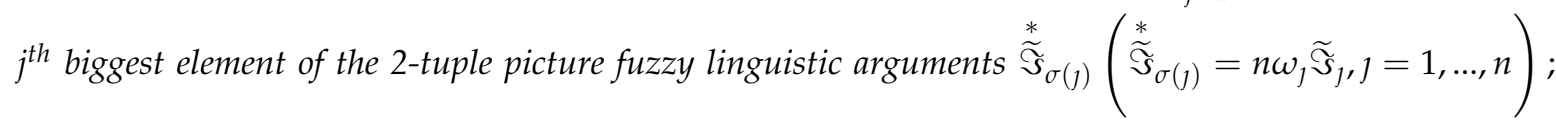
$\left(\omega=\omega_{1}, \ldots, \omega_{n}\right)$ is the weight vector of 2-tuple picture fuzzy linguistic arguments $\widetilde{\Im}_{j}$, with $\omega_{j}>0, \sum_{j=1}^{n} \omega_{j}=1$; and $n$ is the balancing coefficient.

\section{Geometric Aggregation Operators on 2-Tuple Picture Fuzzy Linguistic Numbers}

In this section, we used the information of 2-tuple picture fuzzy linguistic numbers, developed some geometric aggregation operators, and discussed their properties. 
Definition 19. Let $\widetilde{\Im}=\left\langle\left(s_{\ddot{a}}, \alpha\right),\left(s_{\ddot{e}}, \beta\right),\left(s_{\ddot{u}}, \gamma\right)\right\rangle(j=1, \ldots, n)$ be the set of 2-tuple picture fuzzy linguistic numbers. Then, the function of $\Omega^{n} \rightarrow \Omega$ are the 2 -tuple picture fuzzy linguistic weighted geometric (2TPFLWG) operator, defined as

$$
2 T P F L W G_{\Theta}\left(\widetilde{\Im}_{1}, \ldots, \widetilde{\Im}_{n}\right)=\bigotimes_{j=1}^{n}\left(\widetilde{\Im}_{j}\right)^{\Theta_{j}},
$$

where $\Theta=\left(\Theta_{1}, \ldots, \Theta_{n}\right)^{T}$ is the weighting vector of $\widetilde{\Im}_{j}$, such that $\Theta_{j}>0, \sum_{j=1}^{n} \Theta_{j}=1$.

Theorem 2. The aggregated value by using 2TPFLWG operator is also a 2-tuple picture fuzzy linguistic numbers, such that

$$
\begin{aligned}
& 2 \operatorname{TPFLWG} G_{\Theta}\left(\widetilde{\Im}_{1}, \ldots, \widetilde{\Im}_{n}\right)=\bigotimes_{j=1}^{n}\left(\widetilde{\Im}_{j}\right)^{\Theta_{j}}
\end{aligned}
$$

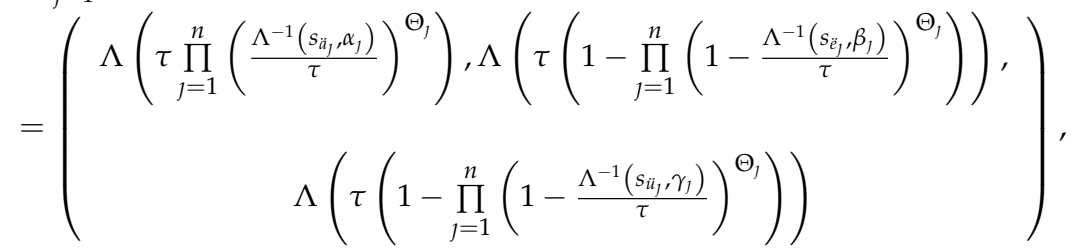

where $\Theta=\left(\Theta_{1}, \ldots, \Theta_{n}\right)^{T}$ is the weights of $\widetilde{\Im}_{j}$, such that $\Theta_{j}>0, \sum_{j=1}^{n} \Theta_{j}=1$.

Proof. We used the mathematical induction principle to prove Equation (28).

(1). When $n=2$, we get

$$
\begin{aligned}
& \left(\widetilde{\Im}_{1}\right)^{\Theta_{1}}=\left(\begin{array}{c}
\Lambda\left(\tau\left(\frac{\Lambda^{-1}\left(s_{\ddot{a}_{1}}, \alpha_{1}\right)}{\tau}\right)^{\Theta_{1}}\right), \Lambda\left(\tau\left(1-\left(1-\frac{\Lambda^{-1}\left(s_{\tilde{s}_{1}}, \beta_{1}\right)}{\tau}\right)^{\Theta_{1}}\right)\right), \\
\Lambda\left(\tau\left(1-\left(1-\frac{\Lambda^{-1}\left(s_{\ddot{u}_{1}}, \gamma_{1}\right)}{\tau}\right)^{\Theta_{1}}\right)\right)
\end{array}\right) . \\
& \left(\widetilde{\Im}_{2}\right)^{\Theta_{2}}=\left(\begin{array}{c}
\Lambda\left(\tau\left(\frac{\Lambda^{-1}\left(s_{\ddot{a}_{1}}, \alpha_{2}\right)}{\tau}\right)^{\Theta_{2}}\right), \Lambda\left(\tau\left(1-\left(1-\frac{\Lambda^{-1}\left(s_{\ddot{s}_{2}}, \beta_{2}\right)}{\tau}\right)^{\Theta_{2}}\right)\right), \\
, \Lambda\left(\tau\left(1-\left(1-\frac{\Lambda^{-1}\left(s_{\tilde{u}_{2}}, \gamma_{2}\right)}{\tau}\right)^{\Theta_{2}}\right)\right)
\end{array}\right) .
\end{aligned}
$$

Then,

$$
\begin{aligned}
& 2 \operatorname{TPFLWG}\left(\widetilde{\Im}_{1}, \widetilde{\Im}_{2}\right)=\left(\widetilde{\Im}_{1}\right)^{\Theta_{1}} \otimes\left(\widetilde{\Im}_{2}\right)^{\Theta_{2}} \\
& =\left(\begin{array}{c}
\Lambda\left(\tau\left(\frac{\Lambda^{-1}\left(s_{\ddot{a}_{1}}, \gamma_{1}\right)}{\tau}\right)^{\Theta_{1}}\left(\frac{\Lambda^{-1}\left(s_{\tilde{a}_{2}}, \gamma_{2}\right)}{\tau}\right)^{\Theta_{2}}\right), \\
\Lambda\left(\tau\left(1-\left(1-\frac{\Lambda^{-1}\left(s_{\dot{e}_{1}}, \alpha_{1}\right)}{\tau}\right)^{\Theta_{1}}\left(1-\frac{\Lambda^{-1}\left(s_{\ddot{e}_{2}}, \alpha_{2}\right)}{\tau}\right)^{\Theta_{2}}\right)\right), \\
\Lambda\left(\tau\left(1-\left(1-\frac{\Lambda^{-1}\left(s_{\dot{u}_{1}}, \alpha_{1}\right)}{\tau}\right)^{\Theta_{1}}\left(1-\frac{\Lambda^{-1}\left(s_{\tilde{u}_{2}}, \alpha_{2}\right)}{\tau}\right)^{\Theta_{2}}\right)\right)
\end{array}\right) .
\end{aligned}
$$


(2). Assume that Equation (28), true for $n=\kappa$; that is,

$$
\begin{aligned}
& 2 \operatorname{TPFLWG} G_{\Theta}\left(\widetilde{\Im}_{1}, \ldots, \widetilde{\Im}_{\kappa}\right)=\bigoplus_{j=1}^{\kappa}\left(\widetilde{\Im}_{j}\right)^{\Theta_{j}} \\
& =\left(\begin{array}{c}
\Lambda\left(\tau \prod_{j=1}^{\kappa}\left(\frac{\Lambda^{-1}\left(s_{\tilde{s}_{\jmath},}, \alpha_{\jmath}\right)}{\tau}\right)^{\Theta_{\jmath}}\right), \Lambda\left(\tau\left(1-\prod_{j=1}^{\kappa}\left(1-\frac{\Lambda^{-1}\left(s_{\ddot{e}_{\jmath}}, \beta_{\jmath}\right)}{\tau}\right)^{\Theta_{\jmath}}\right)\right), \\
\Lambda\left(\tau\left(1-\prod_{j=1}^{\kappa}\left(1-\frac{\Lambda^{-1}\left(s_{\ddot{u}_{\jmath}}, \gamma_{\jmath}\right)}{\tau}\right)^{\Theta_{\jmath}}\right)\right)
\end{array}\right)
\end{aligned}
$$

and we prove Equation (28) for $n=\kappa+1$; then,

$$
\begin{aligned}
& 2 \operatorname{TPFLWG}\left(\widetilde{\Im}_{1}, \ldots, \widetilde{\Im}_{\kappa+1}\right)=\left(\widetilde{\Im}_{1}\right)^{\Theta_{1}} \otimes \ldots \otimes\left(\widetilde{\Im}_{\kappa}\right)^{\Theta_{\kappa}} \otimes\left(\widetilde{\Im}_{\kappa+1}\right)^{\Theta_{\kappa+1}}
\end{aligned}
$$

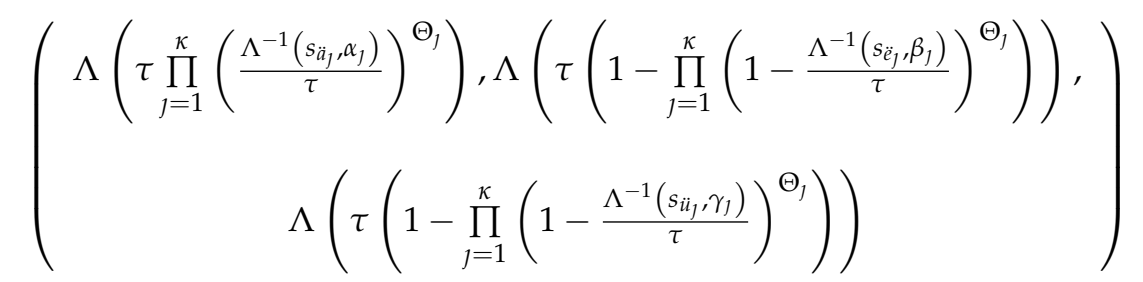

$$
\begin{aligned}
& =\left(\begin{array}{c}
\Lambda\left(\tau\left(\frac{\Lambda^{-1}\left(s_{\ddot{a}_{\kappa+1}}, \alpha_{\kappa+1}\right)}{\tau}\right){ }^{\Theta_{\kappa+1}}\right), \Lambda\left(\tau\left(1-\left(1-\frac{\Lambda^{-1}\left(s_{\ddot{e}_{\kappa+1}}, \beta_{\kappa+1}\right)}{\tau}\right)^{\Theta_{\kappa+1}}\right)\right), \\
\Lambda\left(\tau\left(1-\left(1-\frac{\Lambda^{-1}\left(s_{\tilde{u}_{\kappa+1}}, \gamma_{\kappa+1}\right)}{\tau}\right)^{\Theta_{\kappa+1}}\right)\right)
\end{array}\right) \\
& =\left(\begin{array}{c}
\Lambda\left(\tau \prod_{j=1}^{\kappa+1}\left(\frac{\Lambda^{-1}\left(s_{\ddot{a}_{j},}, \alpha_{\jmath}\right)}{\tau}\right)^{\Theta_{\jmath}}\right), \Lambda\left(\tau\left(1-\prod_{j=1}^{\kappa+1}\left(1-\frac{\Lambda^{-1}\left(s_{\tilde{e}_{\jmath}, \beta_{\jmath}}\right)}{\tau}\right)^{\Theta_{\jmath}}\right)\right), \\
\Lambda\left(\tau\left(1-\prod_{j=1}^{\kappa+1}\left(1-\frac{\Lambda^{-1}\left(s_{i_{j},}, \gamma_{\jmath}\right)}{\tau}\right)^{\Theta_{j}}\right)\right)
\end{array}\right)
\end{aligned}
$$

which shows that the aggregated value is also a 2TPFLN. Therefore, Equation (28) holds for all $n$.

The 2TPFLWG operator have the following properties are satisfied.

Property 4 (Idempotency). If $\widetilde{\Im}_{j}=\widetilde{\Im}$ for all , then

$$
2 \operatorname{TPFLWG}\left(\widetilde{\Im}_{1}, \ldots, \widetilde{\Im}_{n}\right)=\widetilde{\Im} .
$$

Property 5 (Boundedness). Let $\widetilde{\Im}_{j}(j=1, \ldots, n)$ be a set of 2 TPFLNs, and $\widetilde{\Im}^{+}=\max _{j} \widetilde{\Im}_{j}, \widetilde{\Im}^{-}=\min _{j} \widetilde{\Im}_{j}$, then

$$
\widetilde{\Im}^{-} \leq 2 \operatorname{TPFLWG}\left(\widetilde{\Im}_{1}, \ldots, \widetilde{\Im}_{n}\right) \leq \widetilde{\Im}^{+}
$$


Property 6 (Monotonicity). Let $\widetilde{\Im}_{j}(j=1, \ldots, n)$ and $\widetilde{\Im}_{j}^{\prime}(j=1, \ldots, n)$ be a collection of 2 TPFLNs, if $\widetilde{\Im}_{j} \leq$ $\widetilde{\Im}_{j}^{\prime}, \forall j$, then

$$
2 \operatorname{TPFLWG}\left(\widetilde{\Im}_{1}, \ldots, \widetilde{\Im}_{n}\right) \leq 2 \operatorname{TPFLWG}\left(\widetilde{\Im}_{1}^{\prime}, \ldots, \widetilde{\Im}_{n}^{\prime}\right) .
$$

Definition 20. Let $\widetilde{\Im}=\left\langle\left(s_{\ddot{a}}, \alpha\right),\left(s_{\ddot{e}}, \beta\right),\left(s_{\ddot{u}}, \gamma\right)\right\rangle(j=1, \ldots, n)$ be the set of 2-tuple picture fuzzy linguistic numbers. Then, the function of $\Omega^{n} \rightarrow \Omega$ are 2-tuple picture fuzzy linguistic ordered weighted geometric (2TPFLOWG) operator, defined as $\Theta=\left(\Theta_{1}, \ldots, \Theta_{n}\right)^{T}$, which are the associated weights, and $\Theta_{j}>0, \sum_{j=1}^{n} \Theta_{j}=1$. Then,

$$
\begin{aligned}
& 2 \text { TPFLOWG } G_{\Theta}\left(\widetilde{\Im}_{1}, \ldots, \widetilde{\Im}_{n}\right)=\bigotimes_{j=1}^{n}\left(\widetilde{\Im}_{\sigma(\jmath)}\right){ }^{\Theta_{j}} \\
& =\left(\begin{array}{c}
\Lambda\left(\tau \prod_{j=1}^{n}\left(\frac{\Lambda^{-1}\left(s_{\tilde{a}_{\sigma(\jmath)}}, \alpha_{\sigma(\jmath)}\right)}{\tau}\right)^{\Theta_{j}}\right), \Lambda\left(\tau\left(1-\prod_{j=1}^{n}\left(1-\frac{\Lambda^{-1}\left(s_{\tilde{e}_{\sigma(j)}}, \beta_{\sigma(\jmath)}\right)}{\tau}\right)^{\Theta_{j}}\right)\right), \\
\Lambda\left(\tau\left(1-\prod_{j=1}^{n}\left(1-\frac{\Lambda^{-1}\left(s_{i_{\sigma(\jmath)}}, \gamma_{\sigma(\jmath)}\right)}{\tau}\right)^{\Theta_{j}}\right)\right)
\end{array},\right.
\end{aligned}
$$

The permutation of $(1, \ldots, n)$ are $\left(\sigma(1), \ldots, \sigma(n)\right.$, and $\widetilde{\Im}_{\sigma(\jmath-1)} \geq \widetilde{\Im}_{\sigma(\jmath)} \forall \jmath=2, \ldots, n$.

Definition 21. Let $\widetilde{\Im}=\left\langle\left(s_{\ddot{a}}, \alpha\right),\left(s_{\ddot{e}}, \beta\right),\left(s_{\ddot{u}}, \gamma\right)\right\rangle(j=1, \ldots, n)$ be the set of 2-tuple picture fuzzy linguistic numbers. Then, the function of $\Omega^{n} \rightarrow \Omega$ are 2-tuple picture fuzzy linguistic hybrid geometric (2TPFLHG) operator, defined as

$$
\begin{aligned}
& 2 \operatorname{TPFLHG}\left(\widetilde{\Im}_{1}, \ldots, \widetilde{\Im}_{n}\right)=\bigotimes_{j=1}^{n}\left({\underset{\Im}{\widetilde{\Im}}}_{\sigma(\jmath)}^{*}\right)^{\Theta_{j}}
\end{aligned}
$$

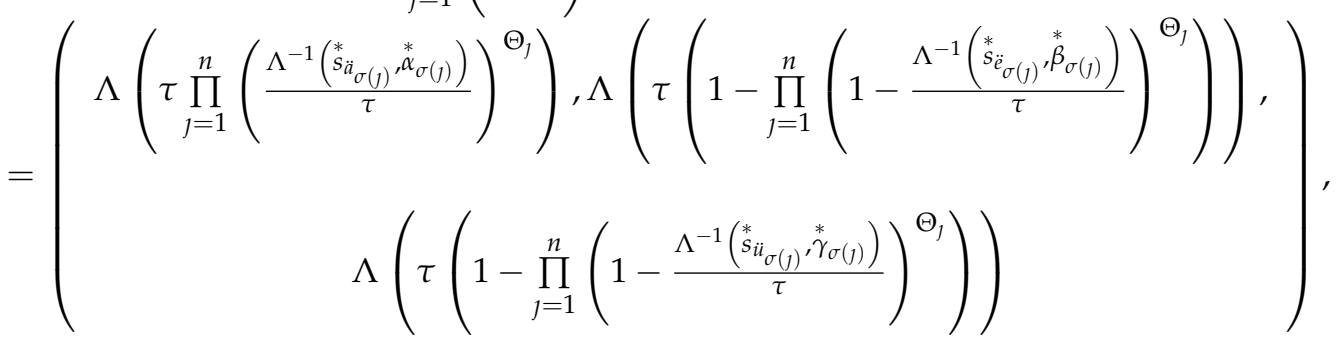

where $\Theta=\left(\Theta_{1}, \ldots, \Theta_{n}\right)^{T}$ are the associated weights, $\Theta_{j}>0, \sum_{j=1}^{n} \Theta_{j}=1$, and $\stackrel{*}{\stackrel{*}{\Im}}{ }_{\sigma(j)}$ is the $j^{\text {th }}$ biggest element of

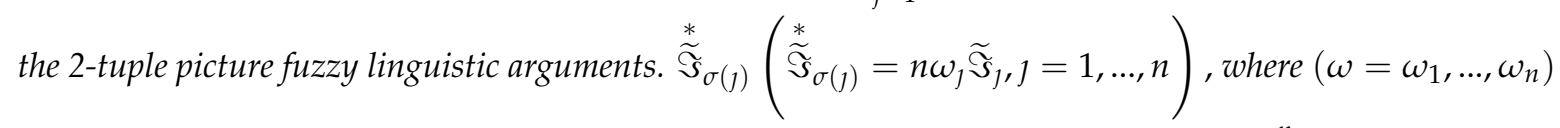
is the weighting vector of 2-tuple picture fuzzy linguistic arguments $\widetilde{\Im}_{j}$, with $\omega_{j}>0, \sum_{j=1}^{n} \omega_{j}=1$, where $n$ denotes the balancing coefficient.

\section{An Approach for MADM with 2-Tuple Picture Fuzzy Linguistic Information}

In this section, based on proposed two operators (2TPFLWA or 2TPFLWG operators), we proposed an approach for MADM problem, with the information of 2-tuple picture fuzzy linguistic information. Suppose that the discrete set of alternatives is $\mathbb{Q}=\left(\mathbb{Q}_{1}, \ldots, \mathbb{Q}_{m}\right)$, and the attributes set is $\mathbb{N}=\left(\mathbb{N}_{1}, \ldots, \mathbb{N}_{n}\right)$, where $\Theta=\left(\Theta_{1}, \ldots, \Theta_{n}\right)^{T}$ is the weights of the attribute $\mathbb{N}_{j}$ and $\Theta_{j} \in[0,1], \sum_{j=1}^{n} \Theta_{j}=1$. Assume that $\mathbb{Z}=\left(\widetilde{r}_{\imath \jmath}\right)_{m \times n}=\left\langle\left(s_{\ddot{u}}, \alpha\right),\left(s_{\ddot{e}}, \beta\right),\left(s_{\ddot{u}}, \gamma\right)\right\rangle_{m \times n}$ is the 2-tuple picture fuzzy linguistic decision matrix, 
where $\widetilde{r}_{l}$, takes the form of the 2-tuple picture fuzzy linguistic numbers, and $\left(s_{\ddot{a}}, \alpha\right),\left(s_{\ddot{e}}, \beta\right),\left(s_{\ddot{u}}, \gamma\right)$ denote the positive, neutral, and negative grades, respectively, such that the alternative $\mathbb{Q}_{1}$ satisfies the attribute $\mathbb{N}_{j}$ given by the decision-makers. Where $\left(s_{\ddot{u}}, \alpha\right),\left(s_{\ddot{e}}, \beta\right),\left(s_{\ddot{u}}, \gamma\right) \in \dot{S}, \alpha_{\imath \jmath}, \beta_{\imath \jmath}, \gamma_{\imath \jmath} \leq[-0.5,0.5)$, $\imath=1, \ldots, m ; \jmath=1, \ldots, n$. Now, we used the 2-tuple picture fuzzy linguistic information and apply the 2TPFLWA or 2TPFLWG operator for the MADM problem.

Step 1: To find the total preference values $\widetilde{\Im}_{l}(l=1, \ldots, m)$ of the alternative $\mathbb{Q}_{j}$, we used the given information of the matrix $\mathbb{Z}$, the 2TPFLWA operator (Equation (21)), and the 2TPFLWG operators (Equation (28)).

$$
\begin{aligned}
& 2 \operatorname{TPFLWA} A_{\Theta}\left(\widetilde{\Im}_{1}, \ldots, \widetilde{\Im}_{n}\right)=\bigoplus_{j=1}^{n}\left(\Theta_{j} \widetilde{\Im}_{\jmath}\right)
\end{aligned}
$$

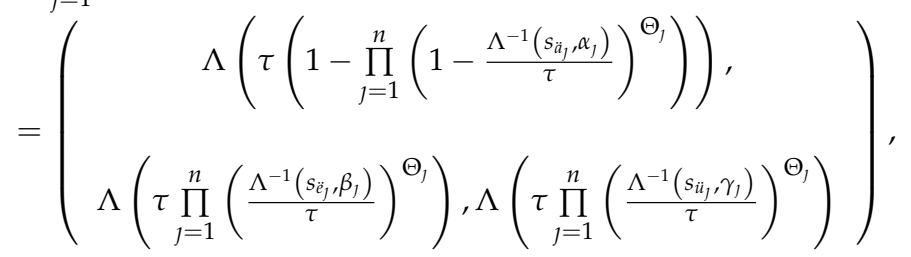

Or

$$
\begin{aligned}
& 2 T P F L W G_{\Theta}\left(\widetilde{\Im}_{1}, \ldots, \widetilde{\Im}_{n}\right)=\bigotimes_{j=1}^{n}\left(\widetilde{\Im}_{j}\right)^{\Theta_{j}}
\end{aligned}
$$

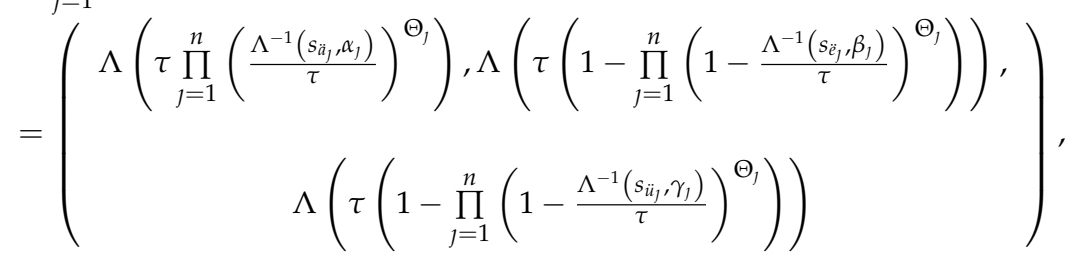

Step 2: Find the scores $\operatorname{Sc}\left(\widetilde{\Im}_{l}\right)(l=1, \ldots, m)$ values, using Equation (18) of the total 2-tuple picture fuzzy linguistic numbers $\widetilde{\Im}_{i}$.

Step 3: According to the score value $S c\left(\widetilde{\Im}_{l}\right)(\imath=1, \ldots, m)$, give ranking to the alternatives $\mathbb{Q}_{l}$ and select the best one.

\subsection{Practical Example}

To demonstrate the application of the developed approach, we present a numerical application about the robot selection by manufacturing unit. A robot can execute instruction on its own, either as a machine or as a virtual intelligent agent. In practice, robots are usually electromechanical machines that are controlled by computers, as well as electronic programming. An industrial robot is an automatically controlled, reprogrammed, multifunctional works and can be programmed on three or more axes. It can be installed permanently or mobile for use in industrial application. It is well known that many industrial robots are present in almost all production or manufacturing industries to improve quality and productivity. Depending on the work performed, choosing the right robot for a critical task has become a challenging task for automated production cells. Various criteria and alternatives are considered to be responsible for the operation of a particular robot. Therefore, to facilitate this evaluation and selection process a strong multicriteria decision support model is indeed required. To address this issue, we present a work that explores the concept of 2TPFLSs, integrated with averaging and geometric operators to help such a decision-making problems.

The given model has been applied for the selection of an industrial robot carried out by three production unit of a famous manufacturing industry in Pakistan. After initial selection, three alternatives robots $\left\{\mathbb{Q}_{1}, \mathbb{Q}_{2}, \mathbb{Q}_{3}\right\}$ were selected for further evaluation. The given set of criteria $\left\{\mathbb{N}_{1}, \mathbb{N}_{2}, \mathbb{N}_{3}, \mathbb{N}_{4}\right\}$ has been considered, where $\mathbb{N}_{1}$ represent the speediness (measure in $\mathrm{m} / \mathrm{s}$, which shows the quickness of the response during the transportation); $\mathbb{N}_{2}$ shows payload capacity (operation limitation or ranged of the robot's payload capacity, measured in $\mathrm{kg}$ ); $\mathbb{N}_{3}$ represent the programming 
flexibility (readability, coordination, and degree of common robotic integration with other robotic system); $\mathbb{N}_{4}$ shows the human-machine interface (capability of easy human-robot interaction). By the existing experience and knowledge, the manufacturing unit uses the criteria weighting vector $\Theta=(0.27,0.24,0.23,0.26)^{T}$. The evaluation matrix is shown in Table 1. In the following, to choose the best robot for the manufacturing unit, we used the proposed approach. The final ranking of robot, see Tables 2-4.

Table 1. The 2-tuple picture fuzzy linguistic decision matrix.

\begin{tabular}{ccccc}
\hline & $\mathbb{N}_{\mathbf{1}}$ & $\mathbb{N}_{\mathbf{2}}$ & $\mathbb{N}_{\mathbf{3}}$ & $\mathbb{N}_{4}$ \\
\hline $\mathbb{Q}_{1}$ & $\left\langle\left(s_{6}, 0\right),\left(s_{1}, 0\right)\left(s_{1}, 0\right\rangle\right.$ & $\left\langle\left(s_{4}, 0\right),\left(s_{2}, 0\right)\left(s_{1}, 0\right\rangle\right.$ & $\left\langle\left(s_{4}, 0\right),\left(s_{2}, 0\right)\left(s_{1}, 0\right\rangle\right.$ & $\left\langle\left(s_{4}, 0\right),\left(s_{2}, 0\right)\left(s_{3}, 0\right\rangle\right.$ \\
$\mathbb{Q}_{2}$ & $\left\langle\left(s_{4}, 0\right),\left(s_{2}, 0\right)\left(s_{2}, 0\right\rangle\right.$ & $\left\langle\left(s_{5}, 0\right),\left(s_{1}, 0\right)\left(s_{2}, 0\right\rangle\right.$ & $\left\langle\left(s_{3}, 0\right),\left(s_{1}, 0\right)\left(s_{2}, 0\right\rangle\right.$ & $\left\langle\left(s_{1}, 0\right),\left(s_{3}, 0\right)\left(s_{4}, 0\right\rangle\right.$ \\
$\mathbb{Q}_{3}$ & $\left\langle\left(s_{5}, 0\right),\left(s_{1}, 0\right)\left(s_{2}, 0\right\rangle\right.$ & $\left\langle\left(s_{4}, 0\right),\left(s_{2}, 0\right)\left(s_{1}, 0\right\rangle\right.$ & $\left\langle\left(s_{1}, 0\right),\left(s_{2}, 0\right)\left(s_{3}, 0\right\rangle\right.$ & $\left\langle\left(s_{2}, 0\right),\left(s_{2}, 0\right)\left(s_{2}, 0\right\rangle\right.$ \\
\hline
\end{tabular}

To choose the most desirable alternative, we used the following steps.

Table 2. The aggregated value of the alternatives by using the 2-tuple picture fuzzy linguistic weighted average (2TPFLWA).

\begin{tabular}{lll}
\hline & 2TPFLWA & 2TPFLWG \\
\hline $\mathbb{Q}_{1}$ & $\left\langle\left(s_{4}, 0.45\right),\left(s_{2},-0.35\right),\left(s_{1}, 0.33\right)\right\rangle$ & $\left\langle\left(s_{4}, 0.44\right),\left(s_{2},-0.25\right),\left(s_{2},-0.42\right)\right\rangle$ \\
$\mathbb{Q}_{2}$ & $\left\langle\left(s_{3}, 0.42\right),\left(s_{2},-0.41\right),\left(s_{2},-0.36\right)\right\rangle$ & $\left\langle\left(s_{3},-0.28\right),\left(s_{2},-0.16\right),\left(s_{3},-0.41\right)\right\rangle$ \\
$\mathbb{Q}_{3}$ & $\left\langle\left(s_{4},-0.28\right),\left(s_{2},-0.39\right),\left(s_{2},-0.18\right)\right\rangle$ & $\left\langle\left(s_{3},-0.46\right),\left(s_{2},-0.19\right),\left(s_{2}, 0.03\right)\right\rangle$ \\
\hline
\end{tabular}

Table 3. Alternatives score values.

\begin{tabular}{lll}
\hline & 2TPFLWA & 2TPFLWG \\
\hline $\mathbb{Q}_{1}$ & $\left(s_{3}, 0.48\right)$ & $\left(s_{3}, 0.38\right)$ \\
$\mathbb{Q}_{2}$ & $\left(s_{3}, 0.06\right)$ & $\left(s_{2}, 0.42\right)$ \\
$\mathbb{Q}_{3}$ & $\left(s_{3}, 0.09\right)$ & $\left(s_{3},-0.44\right)$ \\
\hline
\end{tabular}

Table 4. Ranking of the alternatives.

\begin{tabular}{ll}
\hline Operators & Ranking \\
\hline 2TPFLWA & $\mathbb{Q}_{1}>\mathbb{Q}_{3}>\mathbb{Q}_{2}$ \\
2TPFLWG & $\mathbb{Q}_{1}>\mathbb{Q}_{3}>\mathbb{Q}_{2}$ \\
\hline
\end{tabular}

\subsection{A Comparative Analysis with Linguistic Picture Fuzzy Sets}

The notion of linguistic picture fuzzy set (LPFS) was developed by Zeng et al. [53]. Zeng et al. [53] introduced the extended version of the linguistic picture fuzzy TOPSIS method and solved the problem of enterprise resource planning systems. We solved our created problem, using the concept of Zeng et al. [53].

First, we convert the data of created problem to linguistic picture fuzzy numbers, which are shown in Table 5.

Table 5. The linguistic picture fuzzy decision matrix.

\begin{tabular}{ccccc}
\hline & $\mathbb{N}_{\mathbf{1}}$ & $\mathbb{N}_{\mathbf{2}}$ & $\mathbb{N}_{\mathbf{3}}$ & $\mathbb{N}_{\mathbf{4}}$ \\
\hline $\mathbb{Q}_{1}$ & $\left\langle\left(s_{6}, s_{1}, s_{1}\right)\right\rangle$ & $\left\langle\left(s_{4}, s_{2}, s_{1}\right)\right\rangle$ & $\left\langle\left(s_{4}, s_{2}, s_{1}\right)\right\rangle$ & $\left\langle\left(s_{4}, s_{2}, s_{3}\right)\right\rangle$ \\
$\mathbb{Q}_{2}$ & $\left\langle\left(s_{4}, s_{2}, s_{2}\right)\right\rangle$ & $\left\langle\left(s_{5}, s_{1}, s_{2}\right)\right\rangle$ & $\left\langle\left(s_{3}, s_{1}, s_{2}\right)\right\rangle$ & $\left\langle\left(s_{1}, s_{3}, s_{4}\right)\right\rangle$ \\
$\mathbb{Q}_{3}$ & $\left\langle\left(s_{5}, s_{1}, s_{2}\right)\right\rangle$ & $\left\langle\left(s_{4}, s_{2}, s_{1}\right)\right\rangle$ & $\left\langle\left(s_{1}, s_{2}, s_{3}\right)\right\rangle$ & $\left\langle\left(s_{2}, s_{2}, s_{2}\right)\right\rangle$ \\
\hline
\end{tabular}

Then, we apply all of the steps of the approach of Zeng et al. [53], which, using the weights of the attributes, are $(0.27,0.24,0.23,0.26)^{T}$. We obtain the following ranking. 


\subsection{Comparative Discussion}

To illustrate the effectiveness of the developed algorithm using 2TPFLSs, we give a numerical example and analyze the selection of the best alternative using the developed approach and 2TPFL information. In Table 4, we derived the ranking of the alternatives by utilizing the developed method. From Table 6, we observed that the ranking orders of the alternatives using the developed method are totally matched with the result derived from the Zeng et al. [53] method. Therefore, the proposed approach is also validated. Using the proposed method, the best alternative is $\mathbb{Q}_{1}$, and using the Zeng et al. [53] method, the best alternative is aslo $\mathbb{Q}_{1}$, both are same best alternative $\mathbb{Q}_{1}$ (see Table 6). Thus, the proposed technique is rather prominent, because it can effectively avoid any loss of information which formerly occur during the linguistic information processing. Both numerical and linguistic information are taken into consideration using 2-tuple picture fuzzy linguistic information, which makes the developed techniques more prominent, flexible, and realistic.

Table 6. Ordering of the alternatives.

\begin{tabular}{cc}
\hline Approaches & Ranking \\
\hline LPF TOPSIS Method [53] & $\mathbb{Q}_{1}>\mathbb{Q}_{3}>\mathbb{Q}_{2}$ \\
2TPFLWA operator & $\mathbb{Q}_{1}>\mathbb{Q}_{3}>\mathbb{Q}_{2}$ \\
2TPFLWG operator & $\mathbb{Q}_{1}>\mathbb{Q}_{3}>\mathbb{Q}_{2}$ \\
\hline
\end{tabular}

\section{Conclusions}

In this article, we study a MADM problem with the 2-tuple picture fuzzy linguistic environment. First, we introduced some 2-tuple picture fuzzy linguistic aggregation operators: 2-tuple picture fuzzy linguistic weighted average (2TPFLWA), 2-tuple picture fuzzy linguistic weighted geometric (2TPFLWG), 2-tuple picture fuzzy linguistic ordered weighted average (2TPFLOWA), 2-tuple picture fuzzy linguistic ordered weighted geometric (2TPFLOWG), 2-tuple picture fuzzy linguistic hybrid average (2TPFLHA), and 2tuple picture fuzzy linguistic hybrid geometric (2TPFLHG) operators. We study some basic properties of the defined operators. Then, we use the developed operators and write an algorithm for the solution of MADM problem. The practical application of robot selection by manufacturing unit is given to prove the importance of the proposed method and to establish its practicability and effectiveness. Finally, we compare our proposed method with the Zeng et al. [53] method, to show that our proposed method is more validate, practical, and effective than the other existing methods. In our future work, we will apply the application of 2TPFLNs in many other researches $[22,23,27,30,32]$.

Author Contributions: Project administration, A.A.K.; Conceptualization, Software, Writing-original draft, M.Q.; Writing—review and editing, M.Q. and M.B.; Supervision, S.A.; Funding acquisition, J.L.

Funding: This paper is supported by "Research on the Effectiveness Evaluation, Risk Control and System Construction of the Agricultural Credit Guarantee Policy", the National Natural Science Foundation of China (NSFC), Jan 2019-Dec 2022, No. 71873100. Sponsor and Host: Jianchao Luo. This research work is supported by "Research on the Effect Evaluation, Operational Pattern, Supporting Policy of the Contracted Management of Farmland Mortgage Finance", the National Natural Science Foundation of China (NSFC), Jan 2016. Dec 2019, No. 71573210. Sponsor and Host: Jianchao Luo.

Acknowledgments: The authors would like to thank the Editor in Chief, the Associate Editor, and the anonymous referees for their detailed and valuable comments, which helped improve this manuscript.

Conflicts of Interest: The authors declare no conflict of interest.

\section{References}

1. Zadeh, L.A. Fuzzy sets. Inf. Control 1965, 8, 338-353. [CrossRef]

2. Bellman, R.E.; Zadeh, L.A. Decision-making in a fuzzy environment. Manag. Sci. 1970, 17, B-141. [CrossRef]

3. Buckley, J.J. Fuzzy decision-making with data: Applications to statistics. Fuzzy Sets Syst. 1985, 16, 139-147. 
[CrossRef]

4. Atnassov, K. Intuitionistic Fuzzy Sets: Theory and Applications. Physica-Verlag: Heidelberg, Germany, 1999.

5. Garg, H. Generalized intuitionistic fuzzy interactive geometric interaction operators using Einstein t-norm and t-conorm and their application to decision-making. Comput. Ind. Eng. 2016, 101, 53-69. [CrossRef]

6. Garg, H. Novel intuitionistic fuzzy decision-making method based on an improved operation laws and its application. Eng. Appl. Artif. Intell. 2017, 60, 164-174. [CrossRef]

7. Kaur, G.; Garg, H. Cubic intuitionistic fuzzy aggregation operators. Int. J. Uncertain. Quantif. 2018, 8, 405-427. [CrossRef]

8. Kaur, G.; Garg, H. Generalized cubic intuitionistic fuzzy aggregation operators using t-norm operations and their applications to group decision-making process. Arab. J. Sci. Eng. 2018, 1-20. [CrossRef]

9. Garg, H.; Arora, R. Novel scaled prioritized intuitionistic fuzzy soft interaction averaging aggregation operators and their application to multi criteria decision-making. Eng. Appl. Artif. Intell. 2018, 71C, 100-112. [CrossRef]

10. Garg, H.; Rani, D. Some generalized complex intuitionistic fuzzy aggregation operators and their application to multicriteria decision-making process. Arab. J. Sci. Eng. 2018, 1-20. [CrossRef]

11. Shen, K.W.; Wang, J.Q. Z-VIKOR method based on a new weighted comprehensive distance measure of Z-number and its application. IEEE Trans. Fuzzy Syst. 2018. TFUZZ.2018.2816581. [CrossRef]

12. Wang, X.K.; Peng, H.G.; Wang, J.Q. Hesitant linguistic intuitionistic fuzzy sets and their application in multicriteria decision-making problems. Int. J. Uncertain. Quantif. 2018, 8, 321-341. [CrossRef]

13. Hoskova-Mayerova, S.; Maturo, A. Decision-making process using hyperstructures and fuzzy structures in social sciences. In Soft Computing Applications for Group Decision-making and Consensus Modeling; Springer: Cham, Switzerland, 2018; pp. 103-111.

14. Collan, M.; Kacprzyk, J. (Eds.) Soft Computing Applications for Group Decision-making and Consensus Modeling; Springer International Publishing: Cham, Switzerland, 2018

15. Cuong, B.C. Picture fuzzy sets-first results. In Part 1 "Seminar" Neuro-Fuzzy Systems with Applications; Institute of Mathematics: Hanoi, Vietnam, 2013

16. Cuong, B.C. Picture fuzzy sets-first results. In Part 2 "Seminar" Neuro-Fuzzy Systems with Applications; Institute of Mathematics: Hanoi, Vietnam, 2013

17. Cuong, B.C. Picture fuzzy sets. J. Comput. Sci. Cybern. 2014, 30, 409.

18. Cuong, B.C.; Van Hai, P. Some fuzzy logic operators for picture fuzzy sets. In Proceedings of the 2015 Seventh International Conference on Knowledge and Systems Engineering (KSE), Ho Chi Minh City, Vietnam, 8-10 October 2015; pp. 132-137.

19. Cuong, B.C.; Kreinovitch, V.; Ngan, R.T. A classification of representable t-norm operators for picture fuzzy sets. In Proceedings of the 2016 Eighth International Conference on Knowledge and Systems Engineering (KSE), Hanoi, Vietnam, 6-8 October 2016; pp. 19-24.

20. Phong, P.H.; Hieu, D.T.; Ngan, R.T.; Them, P.T. Some compositions of picture fuzzy relations. In Proceedings of the 7th National Conference on Fundamental and Applied Information Technology Research (FAIR'7), Thai Nguyen, Vietnam, 19-20 June 2014; pp. 19-20.

21. Wei, G.; Alsaadi, F.E.; Hayat, T.; Alsaedi, A. Projection models for multiple attribute decision-making with picture fuzzy information. Int. J. Mach. Learn. Cybern. 2018, 9, 713-719. [CrossRef]

22. Wei, G.; Gao, H. The generalized Dice similarity measures for picture fuzzy sets and their applications. Informatica 2018, 29, 107-124. [CrossRef]

23. Wei, G. Some similarity measures for picture fuzzy sets and their applications. Iran. J. Fuzzy Syst. 2018, 15, 77-89.

24. Singh, P. Correlation coefficients for picture fuzzy sets. J. Intell. Fuzzy Syst. 2015, 28, 591-604.

25. Son, L.H. DPFCM: A novel distributed picture fuzzy clustering method on picture fuzzy sets. Expert Syst. Appl. 2015, 42, 51-66.. [CrossRef]

26. Thong, P.H. A new approach to multi-variable fuzzy forecasting using picture fuzzy clustering and picture fuzzy rule interpolation method. In Knowledge and Systems Engineering; Springer: Cham, Switzerland, 2015; pp. 679-690.

27. Son, L.H. Generalized picture distance measure and applications to picture fuzzy clustering. Appl. Soft Comput. 2016, 46, 284-295. 
[CrossRef]

28. Son, L.H. Measuring analogousness in picture fuzzy sets: From picture distance measures to picture association measures. Fuzzy Optim. Decis. Mak. 2017, 16, 359-378. [CrossRef]

29. Van Viet, P.; Van Hai, P. Picture inference system: A new fuzzy inference system on picture fuzzy set. Appl. Intell. 2017, 46, 652-669.

30. Thong, P.H. Picture fuzzy clustering for complex data. Eng. Appl. Artif. Intell. 2016, 56, 121-130. [CrossRef]

31. Thong, P.H. A novel automatic picture fuzzy clustering method based on particle swarm optimization and picture composite cardinality. Knowl.-Based Syst. 2016, 109, 48-60. [CrossRef]

32. Wei, G. Picture fuzzy cross-entropy for multiple attribute decision-making problems. J. Bus. Econom. Manag. 2016, 17, 491-502. [CrossRef]

33. Yang, Y.; Liang, C.; Ji, S.; Liu, T. Adjustable soft discernibility matrix based on picture fuzzy soft sets and its applications in decision-making. J. Intell. Fuzzy Syst. 2015, 29, 1711-1722. [CrossRef]

34. Garg, H. Some picture fuzzy aggregation operators and their applications to multicriteria decision-making. Arab. J. Sci. Eng. 2017, 42, 5275-5290. [CrossRef]

35. Peng, X.; Dai, J. Algorithm for picture fuzzy multiple attribute decision-making based on new distance measure. Int. J. Uncertain. Quantif. 2017, 7, 177-187. [CrossRef]

36. Phuong, P.T.M.; Thong, P.H. Theoretical analysis of picture fuzzy clustering: Convergence and property. J. Comput. Sci. Cybern. 2018, 34, 17-32. [CrossRef]

37. Thong, P.H.; Fujita, H. Interpolative picture fuzzy rules: A novel forecast method for weather nowcasting. In Proceedings of the 2016 IEEE International Conference on Fuzzy Systems (FUZZ-IEEE), Vancouver, BC, Canada, 24-29 July 2016; pp. 86-93.

38. Van Viet, P.; Chau, H.T.M.; Van Hai, P. Some extensions of membership graphs for picture inference systems. In Proceedings of the 2015 Seventh International Conference on Knowledge and Systems Engineering (KSE), Ho Chi Minh City, Vietnam, 8-10 October 2015; pp. 192-197.

39. Ashraf, S.; Abdullah, S.; Qadir, A. Novel concept of cubic picture fuzzy sets. J. New Theory 2018, 24, 59-72.

40. Herrera, F.; Martinez, L. The 2-tuple linguistic computational model: Advantages of its linguistic description, accuracy and consistency. Int. J. Uncertain. Fuzziness Knowl.-Based Syst. 2001, 9 (Suppl. 1), 33-48. [CrossRef]

41. Herrera, F.; Martınez, L.; Sánchez, P.J. Managing non-homogeneous information in group decision-making. Eur. J. Oper. Res. 2005, 166, 115-132. [CrossRef]

42. Wang, W.P. Evaluating new product development performance by fuzzy linguistic computing. Expert Syst. Appl. 2009, 36, 9759-9766. [CrossRef]

43. Wei, G.W. Extension of TOPSIS method for 2-tuple linguistic multiple attribute group decision-making with incomplete weight information. Knowl. Inf. Syst. 2010, 25, 623-634. [CrossRef]

44. Chang, K.H.; Wen, T.C. A novel efficient approach for DFMEA combining 2-tuple and the OWA operator. Expert Syst. Appl. 2010, 37, 2362-2370. [CrossRef]

45. Jiang, X.P.; Wei, G.W. Some Bonferroni mean operators with 2-tuple linguistic information and their application to multiple attribute decision-making. J. Intell. Fuzzy Syst. 2014, 27, 2153-2162.

46. Li, C.C.; Dong, Y. Multi-attribute group decision making methods with proportional 2-tuple linguistic assessments and weights. Int. J. Comput. Intell. Syst. 2014, 7, 758-770. [CrossRef]

47. Wang, J.Q.; Wang, D.D.; Zhang, H.Y.; Chen, X.H. Multi-criteria group decision-making method based on interval 2-tuple linguistic information and Choquet integral aggregation operators. Soft Comput. 2015, 19, 389-405. [CrossRef]

48. Qin, J.; Liu, X. 2-tuple linguistic Muirhead mean operators for multiple attribute group decision-making and its application to supplier selection. Kybernetes 2016, 45, 2-29. [CrossRef]

49. Zhang, W.; Xu, Y.; Wang, H. A consensus reaching model for 2-tuple linguistic multiple attribute group decision-making with incomplete weight information. Int. J. Syst. Sci. 2016, 47, 389-405. [CrossRef]

50. Herrera, F.; Martínez, L. A 2-tuple fuzzy linguistic representation model for computing with words. IEEE Trans. Fuzzy Syst. 2000, 8, 746-752.

51. Herrera, F.; Martinez, L. An approach for combining linguistic and numerical information based on the 2-tuple fuzzy linguistic representation model in decision-making. Int. J. Uncertain. Fuzziness Knowl.-Based Syst. 2000, 8, 539-562. 
[CrossRef]

52. Cuong, B.C.; Kreinovich, V. December. Picture Fuzzy Sets-a new concept for computational intelligence problems. In Proceedings of the 2013 Third World Congress on Information and Communication Technologies (WICT 2013), Hanoi, Vietnam, 15-18 December 2013; pp. 1-6.

53. Zeng, S.; Qiyas, M.; Arif, M.; Mahmood, T. Extended Version of Linguistic Picture Fuzzy TOPSIS Method and Its Applications in Enterprise Resource Planning Systems. Math. Probl. Eng. 2019, 2019, 8594938. [CrossRef]

(C) 2019 by the authors. Licensee MDPI, Basel, Switzerland. This article is an open access article distributed under the terms and conditions of the Creative Commons Attribution (CC BY) license (http:// creativecommons.org/licenses/by/4.0/). 